\title{
MÁS ALLÁ DE LAS PALABRAS: ¿PUEDE COMPRENDERSE EL GÉNERO DISCURSIVO INFORME DE POLÍTICA MONETARIA DESDE UN ÚNICO SISTEMA SEMIÓTICO PREDOMINANTE? ${ }^{1}$
}

Beyond words: Can the discourse genre Monetary Policy Report be comprehended from a single predominant semiotic system?

Giovanni Parodi*

Cristóbal Julio**

\section{Resumen}

En este artículo investigamos la incidencia de tres formatos de presentación de información multisemiótica textual en la comprensión de textos escritos a partir de un pasaje retórico del género Informe de Política Monetaria (IPOM). Para ello se diseñó un experimento con tres pruebas de comprensión, cuyos textos variaban en la preponderancia multisemiótica constitutiva: (A) texto original, (B) texto con predominancia del sistema gráfico y (C) texto con predominancia del sistema verbal. Estos instrumentos fueron aplicados a 151 estudiantes de una carrera universitaria en el área de economía, Chile, divididos en dos grupos: (1) estudiantes de primer año de universidad y (2) estudiantes de tercer año. Los resultados indican, por un lado, que no existen diferencias estadísticamente significativas entre las tres condiciones de las pruebas de comprensión, en cada grupo de estudiantes (Grupo 1 y Grupo 2). Por otro, sí se registran diferencias significativas entre los resultados de comprensión de los grupos de lectores de primer y tercer año, particularmente a favor de textos que preponderantemente privilegian un solo formato de codificación de la información (verbal o gráfica); mientras que no se distingue por nivel de inserción disciplinar en textos que exigen una lectura integrada de los códigos verbal y gráfico. Se discuten implicancias de estos resultados para la comprensión de textos especializados multisemióticos.

Palabras clave: Informe de Política Monetaria, Comprensión de textos escritos multisemióticos, Géneros discursivos, Multisemiosis, Discurso de la economía.

\section{Abstract}

In this paper we investigate the impact of three presentation formats of textual multisemiotic information in the comprehension of written texts from a rhetorical passage of the genre Monetary Policy Report (MPR). To do this, an experiment with three comprehension tests was designed, in which texts varied in the constituent multisemiotic preponderance: (A) original text, (B) text with predominance of the graphic system, and (C) text with predominance of the verbal system. These instruments were applied to 151 students at a university program in the field of Economics in Chile. They were divided into two groups: (1) university freshmen and (2)

\footnotetext{
${ }^{1}$ Este artículo se enmarca en el Proyecto FONDECYT 1130033. Expresamos un especial agradecimiento a Lisette Figueroa, Bárbara Grandón, Karen Oyarzún y Liliana Vásquez, sin cuyo decidido apoyo en las diversas fases de esta investigación no habríamos podido alcanzar los resultados aquí reportados. Por supuesto que la responsabilidad del contenido del artículo es de los autores.
} 
university third graders. The results indicate, on the one hand, that there are no statistically significant differences between the three conditions of the comprehension tests, in each group of students (Group 1 and Group 2). On the other hand, there are significant differences between the results of the reading comprehension tests of first and third year university students, particularly, in favor of texts predominantly of one information encoding format (verbal or graphic); while there is no difference in terms of the disciplinary university level in texts that required an integrated reading of verbal and graphic codes. Implications of these results for the comprehension of multisemiotic specialized texts are discussed.

Key words: Monetary Policy Report, Comprehension of multisemiotic written texts. Discourse genres, Multisemiosis, Economics discourse.

\section{INTRODUCCIÓN}

La comprensión del discurso escrito ha sido foco de atención y preocupación durante un número significativo de años, tanto desde diversas lenguas maternas como desde las lenguas extranjeras. Ello ha suscitado que un grupo importante de investigadores haya incursionado en diversos estudios que cubren una cantidad relevante de variables asociadas, debido a la complejidad del fenómeno mismo (e.g., van Dijk \& Kintsch, 1983; De Vega, Carreiras, Gutiérrez-Calvo \& Alonso-Quecuty, 1990; Kintsch, 1998; De Vega \& Cuetos, 1999; McNamara \& Magliano, 2009; Alvermann, Unrau \& Ruddell, 2013; Parodi, 2003, 2014; Parodi, Peronard \& Ibáñez, 2010). Estos investigadores provienen de campos multidisciplinares y sus orientaciones han sido, por un lado, más puramente científicas o, por otro, más bien aplicadas o enfocadas a la educación y las didácticas de las lenguas.

Junto a lo anterior, el estudio de la comprensión de textos escritos ha transcurrido desde dominios generales, en temas de la vida cotidiana y, preferentemente, con atención a géneros que involucran modalidades narrativas (e.g., Graesser, Singer \& Trabasso, 1994; Arnoux, 2002; León, 2003) hacia discursos altamente especializados y géneros disciplinares (e.g., Otero, León \& Graesser, 2002; Parodi, 2007; Ibáñez, 2008; Parodi \& Burdiles, 2015). Esto último impone nuevos desafios, ya que implica la constitución de equipos multidisciplinarios en donde el rol del especialista del área se vuelve un factor preponderante en el acercamiento a temas particulares con contenidos altamente técnicos.

Ahora bien, aunque hemos dicho que ha existido una abundante y prolífica investigación en torno a los procesos de comprensión de textos escritos, su foco ha estado primordialmente centrado en el componente verbal de dichos textos. Son, comparativamente, escasos los estudios que atienden a la naturaleza multisemiótica de los textos escritos, ya sea abordando la comprensión de, por ejemplo, gráficos, tablas, diagramas e ilustraciones, o a la comprensión de la interacción entre algunos de estos artefactos multisemióticos y su co-texto verbal. En otras palabras, poco se sabe de cómo se construyen los significados a partir de la información codificada en un texto escrito, 
en donde conjunta y sinérgicamente participan el sistema verbal y otros sistemas semióticos como el pictórico, el matemático, el tipográfico, el del color, y la diagramación (e.g., Lemke, 1998; O’Halloram, 2006; van Leeuwen, 2005, 2006, 2011; Bateman, 2014).

Si bien hace ya algunos años que se presta atención a la comprensión, por ejemplo, de los denominados 'textos ilustrados', como bien revela un estudio de Levie y Lentz (1982) en que se revisa los resultados de 55 experimentos tanto de textos con y sin ilustraciones así como de otros recursos pictóricos, la investigación teórica y empírica a nivel multimodal aún no cuenta con los mismos desarrollos que la centrada en el texto preferentemente verbal. No obstante ello, se detectan algunas líneas enfocadas en el aprendizaje a partir de múltiples sistemas representacionales (e.g., Paivio, 1971, 1986; Sadoski \& Paivio, 2001; Santana, 2010; Pereira \& González, 2011; Aravena, 2011; Holsanova, 2008; Holsanova \& Nord, 2010; Gladic, 2012; Manghi, 2013; Sanchez \& Wiley, 2014; Farías, Obilinovic, Orrego \& Gregersen, 2014; Schüler, Arndt \& Scheiter, 2015), en el aprendizaje multimedial (e.g., Mayer, 2005, 2009; Ainsworth, 2006; Segers, Verhoeven \& Hulstijn, 2008; Rummer, Schweppe, Fürstenberg, Scheiter \& Zindler, 2011; Rau, Michaelis \& Fay, 2015) y, entre otras, en el estudio de la carga cognitiva del procesamiento desde diversos tipos de códigos (e.g., Sweller, 1988; Chandler \& Sweller, 1991; Brüken, Steinbacher, Plass \& Leutner, 2002; Plass, Moreno \& Brünken, 2010; Sweller, Ayres \& Kalyuga, 2011).

En este contexto investigativo es donde el presente estudio se inserta y desde donde buscamos aportar datos empíricos al procesamiento del discurso escrito multisemiótico disciplinar de un género específico. La perspectiva adoptada ha sido la de una investigación basada en corpus, a partir de textos ecológicos y recolectados en contextos de uso cotidiano por comunidades discursivas de especialistas en formación universitaria en la disciplina de economía (Corpus PUCV-UCSC-2013). El objetivo general fue determinar la incidencia, en la comprensión de textos escritos multimodales, de la preponderancia de un sistema semiótico por sobre otro a partir de tres versiones del mismo apartado retórico funcional de un texto del género Informe de Política Monetaria (IPOM). Para cumplir este objetivo se diseñó un experimento con tres pruebas de comprensión, las que se aplicaron proporcional y aleatoriamente a un total de 151 estudiantes universitarios, divididos en dos grupos según nivel de inserción disciplinar (primer y tercer año de una carrera universitaria con especialización en economía). Con las tres versiones del texto se buscaba conocer el grado de comprensión desde, por un lado, la preponderancia del sistema gráfico y, por otro, del sistema verbal, así como también del texto original en su formato integrado de ambos sistemas semióticos.

Esta investigación busca dar respuesta a las siguientes tres preguntas: (1) ¿Es posible comprender un pasaje de un texto escrito del género IPOM desde un solo sistema semiótico predominante? (2) ¿Qué versión del texto resulta en una mejor comprensión textual: (A) la original que integra ambos sistemas semióticos (gráfico y verbal), (B) la predominantemente gráfica, o (C) la predominantemente verbal? y (3) 
¿De qué modo influye el grado de inserción disciplinar (años en una carrera universitaria) en la comprensión de estas tres versiones del texto especializado?

$\mathrm{El}$ artículo se organiza del siguiente modo: en un primer momento describiremos brevemente el marco de referencia en relación con la comprensión de textos escritos multisemióticos. A continuación entregamos antecedentes acerca de los pasos metodológicos para el diseño y elaboración de las tres pruebas de comprensión así como de los procedimientos de evaluación (técnica del resumen) y la muestra de lectores universitarios. Posteriormente, presentamos los resultados obtenidos a partir de los análisis estadísticos. Por último, discutimos los hallazgos y cerramos el trabajo con las conclusiones y las proyecciones para esta línea de investigación.

\section{MARCO DE REFERENCIA}

1.1. Comprensión de textos escritos multisemióticos: tres propuestas para comenzar

Tal como ya veníamos argumentando, todo especialista en comprensión de textos escritos bien sabe que las teorías y los modelos de comprensión se han desarrollado fundamentalmente con foco en el procesamiento del texto escrito estático de rasgos predominantemente verbales (e.g., Parodi, 2003, 2011, 2014). Esto quiere decir que la realidad efectiva de los textos escritos en su naturaleza multisemiótica ha debido enfrentar - comparativamente - un lento y escaso desarrollo tanto teórico como empírico. Muy probablemente, como sostiene Parodi (2014), esto ha ocurrido producto de los tremendos desafios que aún presenta el amplio campo de estudio de la comprensión de textos estáticos eminentemente verbales y de las múltiples incógnitas que aún aguardan exploraciones científicas y respuestas con base empírica desde la perspectiva de solo uno de los diversos sistemas semióticos constitutivos de los textos (véase, De Vega, Graesser \& Glenberg, 2008; Schüler et al., 2015).

Ahora bien, no obstante lo anterior, y como se indicó en el apartado introductorio de este artículo, algunos científicos sí han abordado la reflexión en torno a la comprensión de textos construidos a partir de otros sistemas semióticos, además del verbal. Sin lugar a dudas, los pioneros trabajos de Paivio $(1971,1986,1991)$ y, posteriormente, de Sadoski $(1992,2009)$ y Sadoski y Paivio $(2001,2007)$ destacan por su temprana visión de una teoría que intenta construir - de modo científico y sistemático, como afirman sus autores - un puente entre dos tradiciones de antiguo origen, como son la imaginería mental y el sistema lingüístico o verbal. La Teoría del Código Dual (TCD) — como denominan Paivio y Sadoski a su propuesta - es una teoría acerca de la cognición y de la mente humana. En el núcleo de esta teoría se asume que la cognición opera a partir de dos tipos de representaciones o códigos mentales, los que son cualitativamente diferentes: un código especializado en el lenguaje verbal (logogens) y un código no verbal especializado en el manejo de objetos y eventos no lingüísticos en la forma de imágenes mentales (imagens). Otro supuesto de la TCD es que no existe un código profundo ni abstracto que subyazca o dé cuenta conjuntamente de la información procesada en ambos canales independientes. De este modo, ambos 
sistemas de codificación de información (logogens e imagens) se consideran separados e independientes, aunque se establece que ellos también operan por medio de interconexiones. Esta teoría parte del principio de que ambos sistemas de codificación frecuentemente se emplean en conjunto, aunque los autores declaran que, a veces, un sistema prima por sobre otro en algunas instancias y, en otras, el otro adquiere mayor predominancia.

La TCD ha dado origen o ha sido sustento para un número importante de investigaciones tanto de orden teórico (e.g., Sadoski, Paivio, \& Goetz, 1991; Schnotz, 2002; Mayer, 2005) como empírico (e.g., Sadoski, Willson, Holcomb \& BoulwareGooden, 2005; Sadoski \& Willson, 2006); en particular, destacamos los desarrollos específicos para lectura y escritura (Sadoski \& Paivio, 2001). Además, la TCD también ha sufrido ampliaciones y extensiones. Tal es el caso de su más ambiciosa extensión como explicación de la evolución de la mente, por medio de la cual Paivio (2007) postula el paso filogenético desde una mente no verbal hacia una capaz de procesar información desde diversas fuentes en donde el lenguaje (verbal) se establece como una base que trabaja sinérgica y sofisticadamente con otros sistemas de codificación de información. No obstante el gran impacto y desarrollo de la TCD existen limitaciones y debates en cuanto a sus supuestos y alcances. Básicamente ellos giran en torno a la pregunta de si solo existen estos dos sistemas representacionales (logogens e imagens) en que la mente codifica y representa la información o si podría existir un único formato; $\mathrm{o}$, incluso, si podrían existir otros canales diversos para informaciones de, probablemente, otra naturaleza. Tal como señalamos párrafos más arriba, estos debates se encuentran abiertos y no hay respuestas definitivas (cf. De Vega et al., 2008; Louwerse, 2010; Schüler et al., 2015).

Por su parte, Mayer $(2005,2009,2011)$ también con el objetivo de capturar el procesamiento de más que solo el código verbal ha propuesto la Teoría Cognitiva del Aprendizaje Multimedial (TCAM). Esta teoría descansa sobre tres principios cognitivos fundamentales del aprendizaje: el supuesto del canal-dual, el supuesto de la capacidad cognitiva limitada (cada canal tiene capacidad limitada de procesamiento) y el supuesto del procesamiento activo (el aprendizaje activo implica poner en ejecución conjuntos coordinados de procesos cognitivos). Así, los dos canales de procesamiento codifican inicialmente de forma independiente la información verbal y pictórica; solo luego de una codificación y organización de cada representación se produce una fase de integración. Para la TCAM el aprendizaje ocurre de modo más profundo cuando los seres humanos procesamos conjuntamente palabras e imágenes, que cuando procesamos únicamente palabras. La idea subyacente da origen al denominado Principio Multimedial, esto es, que dos códigos potencian la construcción del significado textual. Al mismo tiempo, esta teoría especifica cinco procesos cognitivos del aprendizaje multimedial: seleccionar palabras, seleccionar imágenes, organizar palabras, organizar imágenes, e integrar. Siguiendo a Mayer (2005, 2009, 2011), estos cinco tipos de procesos cognitivos dan origen a cinco formas de representación para palabras e imágenes en el marco de la TCAM. El nivel crucial de representación lo 
constituye el establecimiento de las conexiones entre las representaciones basadas-enpalabras (word-based) y las representaciones basadas-en-imágenes (image-based), pues —de acuerdo con Mayer $(2005,2009)$ - este paso involucra un cambio desde los dos códigos independientes a un modelo integrado en el que las relaciones y los elementos correspondientes se vinculan unos con otros, desde el input de las representaciones en paralelo. Así, se llega a construir una representación de un modelo integrado que incluye conexiones con el conocimiento previo del lector, operando tanto desde la memoria de largo plazo como desde la memoria de trabajo verbal y visual.

La TCAM puede ser objeto de los mismos cuestionamientos que ha sufrido la TCD de Paivio y Sadoski en cuanto a los tipos de representaciones y los formatos de su codificación, más aún si en la TCAM se postulan cinco tipos de representaciones. No deja de ser una debilidad de esta propuesta, su escasa o nula conexión explícita con otros modelos de comprensión, preferentemente verbales, con gran impacto en la investigación contemporánea, como los trabajos de van Dijk y Kintsch (1983), Kintsch (1998) o McNamara y Magliano (2009), por mencionar solo algunas propuestas relevantes. A pesar de ello, Mayer (2005) sí establece conexiones explícitas con teorías relacionadas como la de Paivio (1986), la de Sweller $(1999,2003)$ y la de Schnotz y Bannert (2003) y Schnotz (2005), todas ellas vinculadas al procesamiento cognitivo de palabras e imágenes.

También sobre la base de los trabajos de Sadoski y Paivio $(2001,2007)$, Schnotz (2002, 2005), Schnotz, Bannert y Seufert (2002), y Schnotz y Bannert (2003), entre otros, han llegado a una propuesta que se denomina Modelo Integrado de Comprensión de Texto e Imagen (MICT\&I). La arquitectura cognitiva del modelo consiste en registros sensoriales, memoria de trabajo y memoria de largo plazo. Incluye, además, un nivel cognitivo (compuesto de dos canales: verbal y pictórico) y otro perceptual (compuesto de múltiples canales sensoriales). El supuesto de base del MICT\&I reside en que los lectores construyen básicamente dos tipos de representaciones: la proveniente del texto (representación proposicional) y la proveniente de la imagen (modelo mental); a ellas, Schnotz y Bannert (2003) denominan representación descriptiva y representación depictiva, respectivamente. El marco de las representaciones duales es explícitamente reconocido por Schnotz (2005) como heredero del concepto bipartito de Paivio (1986) en cuanto a dos códigos mentales diferentes (sistema verbal y sistema de imagen), aunque Schnotz es muy claro al establecer ciertas distinciones y precisiones en favor del modelo integrado, como la construcción de múltiples representaciones a partir de la información a procesar y el procesamiento de dicha información en diversos sistemas mnemónicos.

De acuerdo con Schnotz y Horz (2010), el MICT\&I difiere de la Teoría del Código Dual (Paivio, 1986, 1971) y de la Teoría Cognitiva del Aprendizaje Multimedial (Mayer 2005, 2009) tanto en términos estructurales como en sus predicciones. Básicamente estas diferencias giran en torno a que el MICT\&I asume que no siempre será beneficioso - en términos de aprendizaje - que el texto provea tanto palabras como imágenes, pues se parte del supuesto de que dos códigos no son necesariamente mejor que 
uno. En otras palabras, para la MICT\&I, a diferencia de las otras propuestas, la interacción de diversos sistemas combinados de codificación también puede presentar efectos perjudiciales (Sweller, 2005), bajo determinadas condiciones y para diversos tipos de aprendientes. Uno de estos efectos nocivos es el llamado Efecto General de Redundancia (General Redundancy Effect) (Chandler \& Sweller, 1991). La MICT\&I predice que los aprendientes, con alto grado de experticia y con alto nivel de conocimiento previo en la temática, frecuentemente no requieren procesar textos con palabras e imágenes, es decir, a ellos les basta con textos con información en un solo formato.

Ahora bien, una característica importante de esta propuesta es el modo en que intenta conectarse con trabajos seminales en el ámbito de la comprensión de textos (e.g., van Dijk \& Kintsch, 1983), aunque lamentablemente no rescata los avances más contemporáneos ni adopta una postura crítica respecto del debate en torno a los tipos de representaciones y su formato. También es valioso destacar el modo en que Schnotz (2005) visualiza las limitaciones del modelo y perfila sus posibles desarrollos futuros así como las mejoras de que podría ser objeto. Por su parte, Schontz y Horz (2010), basados en el MICT\&I, profundizan en el aprendizaje multimedial y lo definen sucintamente como la combinación de diferentes formas de representación. Los autores destacan los desarrollos desde los nuevos multimedia e hipermedia y defienden el supuesto de que estos soportes, desde el campo del diseño instruccional, permiten mejor adaptación de la instrucción a las necesidades y preferencias de los aprendientes.

\subsection{La Teoría de la Comunicabilidad: una cuarta propuesta}

Por último, siguiendo con esta revisión teórica, en cuarto lugar nos referiremos a la Teoría de la Comunicabilidad y los supuestos respecto de la comprensión de textos multisemióticos. Al respecto, Parodi $(2011,2014)$ establece que la comprensión de textos escritos constituye un macroproceso multidimensional en el que intervienen múltiples factores de tipo psicosociobiolingüístico y en cuyo núcleo convergen diversos tipos de conocimientos. Por ello, justamente, se postula su carácter multidimensional, pues no es posible explicarla solo por medio de una dimensión o factor único y se hace necesario identificar diversos planos y distinguir dimensiones intrínsecamente vinculadas. En este sentido, Parodi $(2011,2014)$ postula un principio, la Acreditabilidad de lo Comprendido, y tres supuestos centrales distintivos: a) el supuesto de la Cognición Situada, b) el supuesto de la Interactividad, y c) el supuesto de la Socioconstructividad.

De modo sucinto, la Acreditabilidad de lo Comprendido es concebida como un mecanismo psicolingüístico por medio del cual todo lector experto debería ser capaz de dar cuenta de lo leído y comunicarlo por algún medio, siempre y cuando hubiera logrado construir una representación mental coherente de los significados del texto, guiado por sus objetivos de lectura. Este principio de la TC impone un circuito de comunicación en que el lector debe transmitir, por medio de la lengua escrita o de la lengua oral, la construcción que ha realizado de los significados del texto. En este punto, Parodi $(2011,2014)$ aclara que, para cumplir con este principio, es factible que la 
acreditabilidad de lo comprendido se manifieste o realice por medio de otros sistemas semióticos, como una secuencia de dibujos o un esquema o mediante la relación intersemiótica entre más de un sistema semiótico: por ejemplo, verbal y gráfico. Este acto de acreditabilidad se constituye así en revisión de la representación elaborada y en una eventual re-representación o construcción de una nueva representación, que posiblemente ofrezca lugar a una comprensión más plena. Este acto también da cuenta de que la comprensión es un acto social, contextualizado y situado; esto cobra especial relevancia cuando se hace patente que comprender es una actividad guiada por los objetivos de lectura tanto internos del lector (objetivos de lectura propiamente tales), así como por los establecidos por la comunidad o el entorno (objetivos funcionales).

Junto a lo anterior, la TC contempla los diversos sistemas semióticos presentes en los textos escritos estáticos según las diversas disciplinas científicas. Numerosos estudios empíricos dan cuenta de las interesantes variaciones de los artefactos multisemióticos por medio de los géneros especializados disciplinares (e.g., Lemke, 1998; Royce, 1999; Hiippala, 2012; Parodi, 2010; Taboada \& Habel, 2013; Bateman, 2014; Parodi, Boudon \& Julio, 2014; Boudon \& Parodi, 2014). Esta realidad empírica de los rasgos de los textos escritos estáticos impone exigencias de procesamiento divergentes a las cuales se debe atender, si se busca - por ejemplo - apoyar a lectores en el desarrollo de estrategias eficientes en los textos de sus disciplinas. En este contexto, basados en diversos hallazgos empíricos, Parodi $(2010,2014)$ y Parodi, Boudon y Julio (2014) sostienen que todo texto escrito estático está constituido por un conjunto de sistemas semióticos, entre los que — al menos - es posible distinguir los siguientes: el sistema verbal, el sistema gráfico, el sistema matemático, el sistema tipográfico, el sistema del color, y el sistema de la diagramación.

Respecto de los sistemas de codificación mental, la TC propone la existencia de diversos sistemas representacionales, no solo uno verbal y otro gráfico visual. El cerebro humano es capaz de codificar información en formatos de muy variada naturaleza y cuenta con diversos módulos y series de células especializadas que posibilitan el procesamiento de la diversa información contenida en un texto escrito (e.g., van Essen \& Dieker, 2007; Dehaene, 2010, 2011; Koch, 2012). En este sentido, solo dos códigos no son suficientes para describir y explicar la riqueza de la naturaleza de un texto, aunque es evidente que la investigación, entre otros, en biología, neurociencia, neurolingüística y ciencias cognitivas está aún lejos de entregar un detalle de todos los códigos posibles y sus características; una cosa sí es certera y es que el cerebro no tiene un código único, sino muchos (e.g., Dehaene, 2011). Junto con ello se plantea la factibilidad de que no siempre la información que se comunica deba estar integrada por más de un sistema semiótico con el fin de hacerla más eficiente. Como ha demostrado Parodi (2010, 2014), existen géneros discursivos y contextos culturales específicos que revelan que - en determinadas circunstancias - los textos muestran predominancia de un sistema de codificación por sobre otros. Por ejemplo, en el discurso de la filosofía y de la literatura existe predominancia verbal. En ciertos géneros del discurso de la economía existe predominancia de fórmulas matemáticas, gráficos 
estadísticos y tablas. Este tipo de antecedentes empíricos llevan a sostener que no siempre contar con información concurrente desde diversos sistemas producirá un texto integrado de mayor calidad informativa. Para ciertos lectores y en determinados contextos comunicativos, se generarán textos compuestos con predominio de un único sistema sea, por ejemplo, más verbal, más matemático o más gráfico visual.

Asimismo, a la variación constitutiva de los textos según los rasgos del género discursivo, la denominaremos Principio de Predominancia Constitutiva. Este principio apunta a que no siempre es requisito fundamental de comunicación que todo texto se construya desde diversos sistemas semióticos, sino que puede existir predominancia de un sistema por sobre otro, según las demandas comunicativas del entorno, los dispositivos textuales disponibles y los propósitos de los miembros participantes de la comunidad discursiva

Ahora bien, como es fácil comprobar, las propuestas teóricas revisadas para dar cuenta de la comprensión de los textos multisemióticos son aún indagaciones extremadamente preliminares y una mirada profunda y crítica revela que ellas no logran capturar de modo integral la multidimensionalidad del fenómeno del cual buscan dar cuenta. Como ya se ha apuntado, existen limitaciones y cuestionamientos respecto tanto del tipo como al número de códigos o sistemas de representación, así como al formato de estas representaciones, asuntos que también se encuentran en una discusión abierta para el procesamiento solo del sistema verbal.

En este contexto, la distinción, muy en boga en la literatura respectiva, entre texto e imagen como parte de una teoría o modelo de comprensión nos resulta errónea y, como indica Parodi (2010, 2011, 2014), lo que parece estar en discusión es el concepto mismo de texto escrito (estático en este caso). Desde la TC, no es adecuado establecer una distinción entre 'texto e imagen' o entre 'texto e ilustraciones', pues un texto puede potencialmente estar compuesto de diversos sistemas semióticos entre los que pueden estar el verbal y el pictórico, entre varios otros. Por ello, en nuestra opinión, se debe hablar del texto y, si se quiere enfatizar su naturaleza multisemiótica, se debería decir 'el texto y sus sistemas semióticos constitutivos' o alguna expresión similar que dé cuenta de esta concepción.

\section{METODOLOGÍA}

\subsection{El género Informe de Política Monetaria (IPOM)}

El género discursivo IPOM es un tipo de informe especializado preparado por el Banco Central de Chile, el que es publicado cuatro veces al año. Su propósito comunicativo es proyectar la política monetaria del país en cuanto a la inflación a mediano y largo plazo, a partir de la situación vigente en el momento de la elaboración de cada reporte. Se busca así conducir las decisiones en torno a la política monetaria nacional. Su audiencia está definida por ley y está compuesta por el Senado de la República, el Gobierno y el público general. 
El IPOM forma parte de los géneros identificados en el corpus PUCV-UCSC2013 (Parodi, Boudon \& Julio, 2014; Parodi, Julio, Vásquez-Rocca, 2015), el que fue recolectado desde los textos que se registran como lecturas obligatorias y complementarias de las asignaturas pertenecientes al área de economía en dos carreras universitarias (Parodi, Boudon \& Julio, 2014; Boudon \& Parodi, 2014). El IPOM ha sido descrito por Vásquez (2014) como un género en cuya organización retórica y funcional se identifican cinco macromovidas nucleares. Entre ellas, se ha identificado que la macromovida tres, Constatación y Proyección, se reitera tantas veces como el escritor estime conveniente para el desarrollo de los temas abordados, los que presentan cierta autonomía. Es así que esta unidad organizacional cumple una función central en cuanto al propósito comunicativo de los textos de este género (esto es, constatar y proyectar). Dentro de esta macromovida, se ha identificado una movida central que será el núcleo de esta investigación: Panorámica de la Situación Actual y Futura. Por otro lado, en esta macromovida retórica se ha identificado el más alto índice de gráficos y tablas en los textos estudiados de este género (Vásquez, 2014; Parodi et al., 2015).

Debido a la presencia de rasgos provenientes de diversos sistemas y su estatus dentro de la formación disciplinar, el IPOM muestra ricas potencialidades para explorar las variables: a) comprensión de textos con diferencia en la predominancia de un sistema semiótico y b) inserción disciplinar (medida en años de permanencia en la carrera universitaria).

\subsection{Construcción de los instrumentos para el estudio de la comprensión}

2.2.1. Tres versiones de un segmento del IPOM: predominancia de distintos sistemas semióticos

Basados en los trabajos ya mencionados sobre la descripción de la organización retórica del género IPOM, se seleccionó, para la construcción de las pruebas de comprensión, un ejemplar del género publicado en el año 2012 y se extrajo un segmento textual que incluye la movida Panorámica de la Situación Actual y Futura (PSAF), la que es parte de la macromovida Constatación y Proyección. A partir de esto se construyeron dos textos, además del texto fuente original, es decir, se contó con tres textos para la construcción de los instrumentos de comprensión. El objetivo que se buscaba era explorar los procesos de comprensión basados en el principio de predominancia de un sistema semiótico por sobre otro. Así, los tres textos fueron los siguientes: (A) texto original (con interacción de los sistemas semióticos verbal y gráfico), (B) texto con predominancia del sistema gráfico (en esta versión se eliminó, en su mayoría, la participación del sistema verbal), y (C) texto con predominancia del sistema verbal (en esta versión se eliminó la participación del sistema gráfico). Cabe señalar que nos referimos a las versiones $\mathrm{B}$ y $\mathrm{C}$ como 'sistemas predominantes' porque en ninguna se puede aislar por completo un sistema semiótico de otro. A continuación, se presenta la primera página de los tres diferentes formatos de texto. 
Figura 1. Inicio de los textos de las pruebas A, B y C

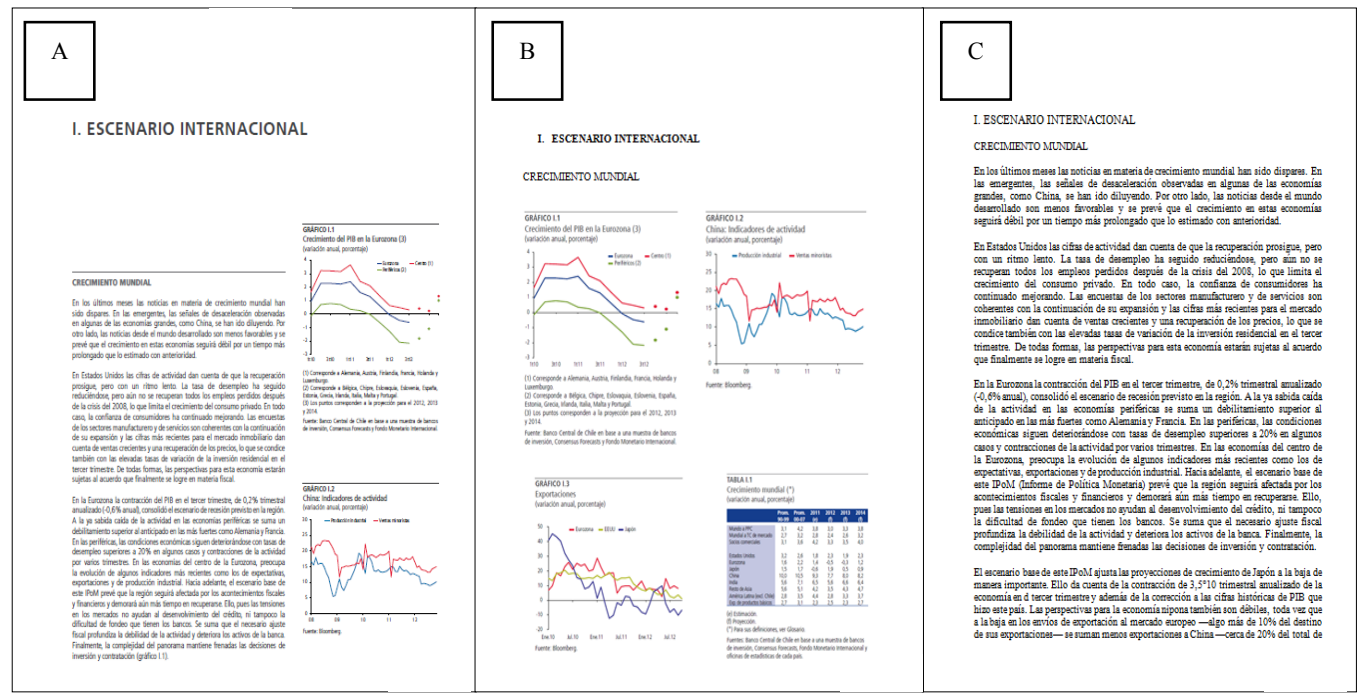

Como se aprecia, en la versión B del texto se dejaron todos los gráficos y la tabla que aparecían en el pasaje textual seleccionado originalmente (compuesto por siete gráficos y una tabla). Se eliminó toda la información verbal que los acompañaba, salvo los títulos y subtítulos de los apartados, las numeraciones y los nombres de los gráficos y tabla, así como las leyendas y notas explicativas. Se respetó el orden de aparición en la organización retórica y se dispusieron los ocho artefactos en una diagramación de doble columna con secuencia de izquierda a derecha y de arriba a abajo. Se buscó así respetar una secuencia de lectura clásica, basada en el flujo de información del texto fuente original. También se respetó el uso del sistema del color en los gráficos y la tabla, provenientes del texto fuente, al constituir este sistema un elemento clave en la construcción de significados.

Por su parte, en la versión predominantemente verbal, es decir, la denominada C, se procedió a eliminar los ocho artefactos ya identificados y se estructuró la página con márgenes de la denominada 'configuración normal'. Se respetaron los títulos y subtítulos originales y se dejó interlineado doble entre párrafo y párrafo. Asimismo, se eliminó toda referencia explícita a gráficos y tabla. Respecto de la versión original, la denominada A, como se muestra en la Figura 1, el texto se mantuvo en su formato fuente y no se introdujo ninguna variación.

2.2.2. Comprensión multimodal del género IPOM: el resumen y la pauta de corrección

Estos tres textos fueron usados como insumos para la elaboración de tres instrumentos de estudio de la comprensión, configurándose, de este modo, tres formatos de prueba de acuerdo con el texto fuente de cada una (A-B-C). Para conocer la 
influencia del formato de representación de la información textual en la comprensión de los estudiantes, se utilizó la técnica del resumen (Parodi, Ibáñez \& Venegas, 2014). El resumen fue requerido mediante una tarea de lectura idéntica para los tres formatos de prueba. La tarea de lectura, que instruyó y dio contexto a la escritura del resumen, fue la siguiente:

"Con el fin de informar a un grupo de profesores de economía de modo resumido el contenido de un documento escrito, te pedimos que leas detenidamente el siguiente texto y luego escribas un resumen que dé cuenta de las ideas nucleares allí presentes. No puedes usar más del espacio que se indica en el cuadro de respuesta. Si te parece, puedes tomar notas para orientar tu resumen con los materiales que aquí te dejamos. También, si lo deseas, puedes escribir o hacer marcas en el texto de lectura."

De esta forma, a la tarea de resumir se le otorgó un objetivo con foco en las ideas nucleares del texto y, además, se les asignó un espacio específico (10 líneas) para transmitir el contenido central de cada texto. También se especificó un tiempo máximo de 90 minutos para completar la tarea.

El proceso psicolingüístico de resumir un texto por escrito implica una serie de actividades lingüísticas y cognitivas. Según Parodi, Ibáñez y Venegas (2014), la actividad de dar cuenta de lo comprendido de modo sucinto puede materializarse mediante diversos modos semióticos, como un gráfico o un diagrama o en palabras. Desde este enfoque es relevante destacar que se concibe al sujeto como un lector/escritor quien debe ser capaz de ejecutar ambos procesos psicolingüísticos como parte de una misma macroactividad, es decir, debe leer y escribir. Además, tanto el texto fuente como el resumen producido pueden manifestarse desde una o varias combinaciones de sistemas semióticos y en distintos niveles de abstracción de la información codificada. Es la macroactividad de lectura o, en este caso, la tarea de lectura, la que orienta al resumen, indicando al lector/escritor de qué forma debe hacer su tarea.

Respecto del procedimiento de revisión y puntaje otorgado a los resúmenes elaborados por los estudiantes, se pidió —en un primer momento - a tres expertos del área de economía que elaboraran un resumen a partir de cada versión de los tres tipos de texto fuente ya mencionados, dando cuenta de las ideas principales contenidas en cada texto. Posteriormente, estos resúmenes expertos fueron contrastados con los pasos retóricos descritos en la investigación de Vásquez (2014) para el género IPOM. Se constató que los pasos, descritos por Vásquez (2014), correspondían con las ideas principales expresadas en sus resúmenes por los expertos a partir de los tres formatos del texto. La pauta de puntaje elaborada sobre estos antecedentes se presenta en la Tabla 1. 
Tabla 1. Puntajes para la revisión de los resúmenes

\begin{tabular}{|c|c|c|c|}
\hline \multicolumn{4}{|c|}{ Pauta de Puntajes } \\
\hline Movida & Paso & Puntos por paso & Porcentaje \\
\hline \multirow{3}{*}{$\begin{array}{c}\text { Panorámica de la } \\
\text { Situación Actual y }\end{array}$ Futura (PSAF) } & Constatación 1 & 10 & 25 \\
\cline { 2 - 3 } & Evidencia 1 & 5 & \\
\cline { 2 - 3 } & Constatación 2 & 10 & 25 \\
\cline { 2 - 3 } & Evidencia 2 & 5 & 50 \\
\cline { 2 - 3 } & Proyección & 30 & 100 \\
\hline \multicolumn{2}{|r}{ TOTALES } & 60 & \\
\hline
\end{tabular}

Como lo muestra la Tabla 1, se optó por un puntaje diferencial basado en la relevancia funcional que cada segmento textual cumple en función del segmento mayor que lo contiene. De esta forma, se consideró la función que un paso retórico cumple dentro de la movida retórica, así como la movida dentro de la macromovida y, por consiguiente, la macromovida dentro del género. Por último, con el fin de buscar la confiabilidad de la revisión y puntuación de los resúmenes, se optó por un diseño de observación cruzada en el que cada resumen era analizado por dos analistas independientes, previamente entrenados en los rasgos retórico funcionales del IPOM, en sus características multimodales y en el uso de la pauta de puntuación. Si se registraba un acuerdo inferior al $80 \%$ entre ambos revisores, se procedía a incorporar un tercer revisor para dirimir la diferencia.

\subsection{La muestra: estudiantes universitarios del área de economía}

Siguiendo los objetivos de esta investigación, se procedió a contactar a un grupo de estudiantes de una carrera ligada al área de economía de una universidad chilena. Buscábamos acceso a dos grupos de estudiantes de distintos niveles de inserción disciplinar, medida por los años de permanencia en la carrera. De este modo se constituyó un grupo de 151 estudiantes, los que se distribuyeron entre ler y 3er año. De manera de mostrar la distribución de la muestra de los sujetos lectores y las respectivas pruebas que rindieron, se elaboró la siguiente Tabla 2:

Tabla 2. Distribución de la muestra de estudiantes

\begin{tabular}{|c|c|c|c|c|}
\hline Inserción disciplinar & \multicolumn{3}{|c|}{ Tipo de Prueba/ $\mathrm{N}^{\circ}$ de sujetos } & \multirow{2}{*}{ TOTAL } \\
\hline \multirow{2}{*}{ 1er año } & Completa (A1) & Gráfico (B1) & Verbal (C1) & \multirow{2}{*}{66} \\
\cline { 2 - 4 } & 22 & 22 & 22 & \\
\hline \multirow{2}{*}{3 3er año } & Completa (A2) & Gráfico (B2) & Verbal (C2) & \multirow{2}{*}{85} \\
\cline { 2 - 4 } & 29 & 28 & 28 & 151 \\
\hline
\end{tabular}

Como se ha señalado, en la Tabla 2 se presentan los dos grupos de estudiantes por año en la carrera y, también, por tipo de prueba que rindieron. Las pruebas fueron repartidas aleatoriamente entre los sujetos, cuidando de tener una cantidad equilibrada de los tipos de prueba en cada grupo. Los datos de la Tabla 2 permiten ilustrar de modo 
resumido las variables en estudio en esta investigación: tipo de prueba según predominancia de sistema semiótico en un género del discurso de la economía (tres tipos: A-B-C) y grado de inserción disciplinar en la carrera universitaria (dos niveles: 1 y 2). Como se mencionó anteriormente, la variable respuesta corresponde al puntaje obtenido en el resumen realizado por los estudiantes. Estas variables son congruentes con el objetivo de la investigación y las respectivas preguntas y serán las que se tomarán en cuenta para los cálculos estadísticos; del mismo modo, ellas guiarán el análisis de resultados.

\subsection{Las hipótesis}

Las preguntas de investigación que guían este estudio nos llevaron a plantear las siguientes hipótesis:

Hipótesis 1: Para cada grupo (primer y tercer año), los puntajes de cada tipo de prueba presentan diferencias estadísticamente significativas.

Hipótesis 2: Entre los dos grupos de lectores (primer y tercer año), los puntajes son estadísticamente diferentes en cada uno de los tipos de pruebas.

\section{RESULTADOS Y DISCUSIÓN}

\subsection{Pruebas estadísticas}

Los resultados de la prueba estadística no paramétrica (Kruskal-Wallis) muestran que no se registró diferencias estadísticamente significativas (valor- $\mathrm{p}=0,589$; valor- $\mathrm{p}=$ 0,076 ) en los resultados de comprensión entre los tres tipos de instrumentos aplicados al total de sujetos encuestados (151 universitarios), según grupo de estudiantes, con un nivel de significancia del $5 \%$. Esto quiere decir que, como muestra la Tabla 4, no existe evidente muestral suficiente para suponer que las tres condiciones de las pruebas de comprensión de un segmento retórico del género IPOM en el discurso de la economía son diferentes, ejecutadas tanto por estudiantes de primer año como de tercer año.

Tabla 3. Comparación de los resultados de los tres formatos de prueba por grupo de estudiantes

\begin{tabular}{|c|c|}
\hline Tres pruebas / Inserción disciplinar & valor-p \\
\hline Tres pruebas estudiantes Primer año & 0,589 \\
\hline Tres pruebas estudiantes Tercer año & 0,076 \\
\hline
\end{tabular}

En este contexto no existe evidencia muestral que apoye la Hipótesis 1, para cada grupo, de esta investigación. Así, basados en estos datos estadísticos, cabe señalar que no importaría el formato de la representación de la información del texto para alcanzar un grado de comprensión de un texto especializado, sea este predominantemente gráfico, predominantemente verbal o en la versión original integrada por ambos sistemas, al interior de un mismo grupo de lectores. Según estos resultados, por cada nivel de inserción disciplinar, los estudiantes de la muestra comprenden de forma similar la información entregada, sin importar el tipo de formato 
en que el texto se encuentre (gráfico, verbal o integrado). Esto indica que el texto fuente original integrado por ambos sistemas semióticos ofrece una reiteración en la información presentada entre el sistema verbal y el gráfico, pues al eliminar uno de los sistemas semióticos, los estudiantes logran construir resúmenes que no se diferencian estadísticamente entre sí. Este hecho demuestra que los resúmenes son de similar calidad y que los lectores logran recuperar información semejante a partir de textos de naturaleza diferente en su formato, pero que semánticamente serían muy similares. También se desprende que los lectores de la muestra por cada nivel universitario poseen habilidades para 'leer y comprender' información textual en formatos diferentes, ya que escriben resúmenes que recuperan contenidos semánticos similares.

En este sentido, respecto de la pregunta de investigación planteada al inicio de este artículo con relación a si es posible comprender un texto desde un solo sistema preponderante, podemos decir que efectivamente se ha demostrado que sí es factible al no existir diferencia estadísticamente significativa en los resultados de comprensión entre los tipos de prueba.

Ahora bien, estos datos aportan evidencia, presumiblemente contraria al llamado Principio Multimedial (Mayer, 2005, 2009, 2011; Fletcher \& Tobias, 2005), en el que se establece que los aprendientes logran mejores aprendizajes a partir de textos que incluyen conjuntamente el sistema verbal y del sistema gráfico visual, no así cuando la información textual se presenta exclusivamente con el sistema verbal. Al respecto, Fletcher y Tobias $(2005)$ y Mayer $(2009,2011)$ enfatizan que existe una importante cantidad de estudios empíricos que han probado la predicción de este principio, aunque también aclaran que, para sujetos con alto conocimiento previo en el dominio, la presencia del sistema gráfico aporta pocos beneficios. No obstante esto último, este principio destaca la confluencia de diversos sistemas en favor de aprendizajes de mejor calidad; hecho que —en este caso - nuestros hallazgos no estarían apoyando.

En lo que sigue se entregan los resultados correspondientes a la segunda hipótesis de esta investigación, para los que se aplicó la prueba no paramétrica MannWhitney, con un nivel de significancia del $5 \%$.

Tabla 4. Resultados en los tres tipos de prueba y los dos grupos de estudiantes

\begin{tabular}{|l|c|}
\hline \multicolumn{1}{|c|}{ Tipo de prueba y grupo de lectores } & valor $-\mathrm{p}$ \\
\hline Prueba A (versión completa integrada) en estudiantes de 1er y de 3er año & 0,6826 \\
\hline Prueba B (predominantemente gráfica) en estudiantes de 1er y de 3er año & 0,0105 \\
\hline Prueba C (predominantemente verbal) en estudiantes de 1er y de 3er año & 0,0057 \\
\hline
\end{tabular}

Según se aprecia en la Tabla 4, los resultados de la prueba estadística no paramétrica muestran que no se registró diferencia estadísticamente significativa (valor$\mathrm{p}=0,6826$ ) para la comparación entre los dos grupos de lectores (ler y 3er año) en la versión completa original de la prueba de comprensión (Prueba A). Sin embargo, sí se observaron diferencias significativas entre los dos grupos de estudiantes tanto en los formatos de representación predominantemente gráfico (Prueba B) como verbal 
(Prueba C) (valor-p $=0,0105$ y valor-p $=0,0057$, respectivamente), a favor de los estudiantes de curso superior.

En este contexto, solo en el primer caso no existe evidencia muestral suficiente para apoyar la hipótesis; por lo tanto, no existe razón para creer que haya diferencia en la versión completa integrada de la prueba entre primer año y curso superior. Para los otros dos casos sí existe evidencia que permite apoyar la Hipótesis 2; por lo tanto, no existe razón para creer que haya diferencia en la versión completa integrada de la prueba entre primer año y curso superior. Para los otros dos casos sí existe evidencia muestral suficiente que permite apoyar la Hipótesis 2, es decir, las diferencias entre los puntajes de las pruebas respectivas son estadísticamente distinguibles.

Esto quiere decir que, para los estudiantes de curso superior con mayor conocimiento previo del tema, del género, de los diferentes sistemas semióticos y de los artefactos gráficos, no se registró una diferencia estadísticamente significativa respecto de los estudiantes de primer año en la lectura de la Prueba A. Como se sabe, este género constituye parte de las lecturas académicas en las carreras en formación universitaria en la disciplina de economía (Corpus PUCV-UCSC-2013) y cumple un rol importante en la construcción de conocimientos especializados. Las razones de esta igualdad de condiciones ante una misma condición de prueba de comprensión no habrá que buscarlas en la variable inserción disciplinar.

Por su parte, los datos de la Tabla 4 indican que las pruebas B y C sí registran diferencias a favor de los estudiantes de curso superior. Esto quiere decir que los estudiantes con mayor inserción disciplinar logran llevar a cabo de mejor modo la tarea del resumen tanto en la versión gráfica como en la versión verbal, hecho que muestra que los lectores con mayor conocimiento disciplinar de economía y del género IPOM logran comprender un texto en una versión construida a partir de un único sistema semiótico preponderante: gráficos o palabras.

En este sentido, los resultados indican que los estudiantes con mayor nivel de inserción disciplinar logran comprender la información codificada únicamente por medio de los gráficos (Prueba B) y realizar un resumen que dé cuenta del contenido semántico central del texto, a diferencia de los estudiantes principiantes en la disciplina de economía. Estos hallazgos indican que leer gráficos es una habilidad que se aprende a lo largo de los años de universidad al interior de una disciplina y también al ir desarrollando grados de conocimiento de géneros especializados, como el IPOM. Por otro lado, estos hallazgos se alinean con la idea de Schnotz respecto de que no siempre la confluencia de información desde diversos sistemas o códigos sea necesariamente un aporte positivo a la mejor y más profunda comprensión. Al respecto, cabe recordar el denominado Efecto General de Redundancia (Schnotz, 2005; Schnotz \& Horz, 2010; Sweller, 2005; Sweller, Ayres \& Kalyuga, 2011; Leahy \& Sweller, 2011), bajo el cual los aprendientes con alto grado de conocimiento previo, se desempeñan mejor en tareas de comprensión con textos en que predomina un solo sistema semiótico. Desde la perspectiva de este efecto, el procesamiento de textos con información proveniente desde dos o más sistemas semióticos, que construyan un texto redundante, produce una 
sobrecarga cognitiva en la memoria de trabajo y, consecuentemente, un impedimento en un mejor procesamiento en la memoria de largo plazo. En efecto, Schnotz (2005) en su modelo integrado predice que los lectores con mayor bagaje de conocimientos obtendrán mejores logros a partir de textos con información desde una sola fuente (solo gráfico o solo palabras). Por su parte, Sweller (2005) y Sweller, Ayres y Kalyuga (2011) aportan diversas evidencias empíricas que demuestran que —en muchos casos - las versiones en formato solo gráfico obtuvieron los mejores resultados de aprendizaje, a diferencia de las versiones más convencionales que requerían integración entre lo verbal y lo gráfico visual, las que resultaron menos efectivas.

Respecto de la Prueba C (predominantemente verbal), según los resultados estadísticos obtenidos, se aplica lo dicho con anterioridad para la Prueba B en cuanto al Efecto General de Redundancia. Esto quiere decir que, cuando los aprendientes tienen alto conocimiento previo, frecuentemente no requieren textos con información integrada, sino que solo en un formato; en este caso, sería el verbal. En este sentido, este hallazgo empírico a favor del formato solo verbal y en detrimento del completo integrado (Prueba A) es coherente con la idea de que:

“... adding a picture to a written text or adding written text to a picture means adding unneeded information. Although, only one of the two information sources is needed, the eye wonders between both of them resulting in a split of attention. Thus, the learner loses time and expends mental effort searching for redundant information." (Schnotz \& Horz, 2010:147)2 .

Lo anterior podría implicar que los lectores que enfrentan la versión completa del segmento textual del IPOM: a) no invierten el tiempo de manera eficiente para leer el texto completo y realizar el resumen, b) emplean sus recursos cognitivos en integrar al resumen solo lo que han alcanzado a leer, y c) si lo han leído completo, no alcanzan a construir una representación mental de la información textual suficientemente coherente, ya que, probablemente, no logran integrar toda la información disponible. Todo ello a diferencia de lo que acontece para los lectores que leen las versiones gráfica y verbal. Ahora bien, retomando las últimas preguntas de investigación, para un grupo de lectores de la muestra, sí es posible comprender textos de economía desde un único sistema semiótico preponderante (ya sea verbal o gráfico), pero cuando se cuenta con un grado mayor de inserción disciplinar, como lo muestran los hallazgos reportados para los estudiantes de tercer año de universidad.

\footnotetext{
2 “....agregar una imagen a un texto escrito o agregar texto escrito a una imagen significa agregar información innecesaria. Aunque solo una de las dos fuentes de información es necesaria, el ojo deambula entre estas dos lo que resulta en una división de la atención. Por lo tanto, el aprendiente pierde tiempo y gasta esfuerzo mental al buscar información redundante" (Trad., nuestra).
} 


\subsection{Logros según organización retórica del IPOM}

A continuación, se presenta la distribución porcentual de los logros en torno a la comprensión de los pasos retóricos evaluados en el total de la muestra de sujetos, con el propósito de estudiar posibles diferencias según tipo de prueba y grupo de estudiantes.

Gráfico 1. Comparación logros en comprensión por tipo de prueba

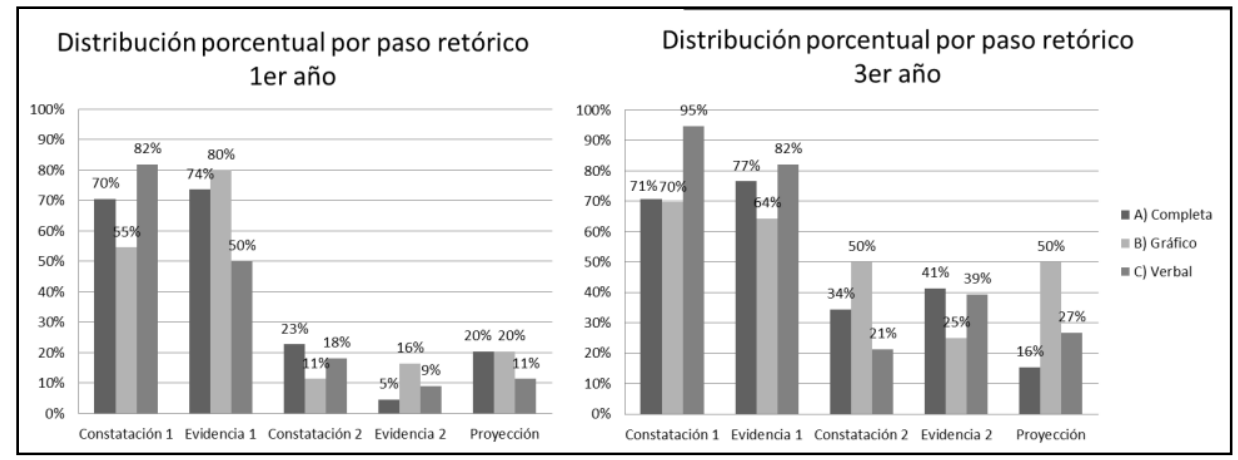

Como se puede apreciar en el Gráfico 1, a partir del análisis pormenorizado de los resúmenes, se constata un mayor logro general promedio en la comprensión de los pasos retóricos del grupo de lectores de tercer año. También vale la pena destacar una cierta regularidad en los pasos con mayor porcentaje de logro, ya que en ambos grupos de estudiantes y en los tres tipos de prueba, los pasos con mayor porcentaje evidenciado son Constatación 1 y Evidencia 1. Esto muestra que los lectores, al construir los resúmenes, se centraron, en general, en los primeros apartados del texto, decayendo de manera progresiva la comprensión de los pasos posteriores. En definitiva, es evidente que se tendió a comprender y, posteriormente, a resumir — preferentemente - la primera parte del texto. Como ya se había señalado en el apartado anterior, los estudiantes de curso superior - en promedio general de todas las pruebas - son notoriamente mejores comprendedores $(51 \%)$ que los novatos de primer año $(36 \%)$, aunque los porcentajes de logro alcanzados en muchos de los resultados parciales tampoco indican un dominio avanzado de los procesos de comprensión textual. Aplicada la prueba no paramétrica Mann-Whitney con un nivel de significancia del 5\%, existe evidencia muestral suficiente para decir que los puntajes de las pruebas de primer año son menores que los puntajes de las pruebas de tercer año (valor-p $=0,0006)$. A la luz de estos datos se reitera la relevancia de la variable inserción disciplinar como un componente que efectivamente logra apoyar el desarrollo de la capacidad de comprensión de textos escritos en todos los formatos en estudio.

Otro aspecto importante a considerar es el relativamente alto porcentaje de logro en el paso retórico Proyección en la Prueba B (versión gráfica) de estudiantes de tercer año $(50 \%)$, en comparación con el grupo de primer año (20\%), así como también con 
las demás pruebas de su mismo grupo. Aplicada la prueba no paramétrica MannWhitney con un nivel de significancia del 5\%, existe evidencia muestral suficiente para decir que los puntajes de la Proyección de la prueba B realizada por alumnos de tercer año son mayores que los puntajes de la Proyección de la prueba B realizada por alumnos de primer año (valor-p $=0.0290$ ). La Proyección, como se indicó anteriormente, constituye el paso retórico fundamental dentro del segmento retórico seleccionado del género IPOM, ya que aporta de modo central al establecimiento del propósito comunicativo del género; por ello, se le asignó el mayor puntaje en todas las versiones de las pruebas. Resulta especialmente interesante notar cómo, en las pruebas con predominancia del sistema gráfico, los estudiantes de tercer año lograron leer esta información en los textos, la comprendieron y, luego, la verbalizaron en los resúmenes. Ello adquiere particular significado si se tiene en cuenta que, en los gráficos, la Proyección estaba consignada de manera representacional por medio de un plano cartesiano, por lo que para construir una representación cognitiva de esta información se hacía necesario recodificar los significados presentados gráficamente con el fin de explicitarlos de manera verbal en el formato que el resumen exigía. Este proceso de comunicabilidad de lo leído se enmarca en el circuito de comunicación establecido en la Teoría de la Comunicabilidad (Parodi, 2014), así como en el proceso de resumir verbalmente por escrito, como lo detallan Parodi, Ibáñez y Venegas (2014).

\section{CONCLUSIONES}

Esta investigación ha aportado evidencia que permite establecer una relación positiva entre la predominancia de un único sistema semiótico, como el verbal o el gráfico, la comprensión de textos escritos especializados en economía y el nivel de inserción disciplinar. Mientras no se detectan diferencias estadísticamente significativas en comprensión entre las tres pruebas por cada grupo de estudiantes de primer y tercer año por separado, sí se encontró que los estudiantes de curso superior comprenden mejor la versión predominantemente verbal y la predominantemente gráfica; aunque la versión completa e integrada por ambos sistemas demostró no arrojar diferencias entre grupos de lectores por estrato universitario. En este sentido, a la luz de la Teoría de la Comunicabilidad (TC), los hallazgos aportados indican que es posible predecir que un lector/escritor con adecuado dominio disciplinar puede comprender un texto predominantemente gráfico, construir una representación cognitiva coherente de esos significados textuales en uno o más tipos de códigos mentales y, luego, orientado por una tarea de escritura, llegar a producir un resumen en un código básicamente verbal, llevando así a cabo una sincronización de diversos sistemas representacionales y logrando transformar información desde unos códigos a otros. En otras palabras, desde los principios rectores de la TC, los lectores/escritores de curso superior en el área de economía de un pasaje retórico del género IPOM establecen un circuito comunicacional en que van desde el texto fuente en versión gráfica hasta la escritura de un resumen en 
versión verbal, pasando por la construcción de una representación cognitiva en probablemente dos códigos: uno gráfico y otro verbal.

De modo más específico, el desarrollo de un cuerpo de conocimientos disciplinares en economía, el manejo del discurso especializado escrito y sus rasgos multisemióticos y el aprendizaje del sistema de gráficos estadísticos de la especialidad establece una distinción fundamental entre los lectores de primer y tercer año, particularmente a favor de textos que preponderantemente privilegian un solo formato de codificación de la información (verbal o gráfica). Por el contrario, los textos que exigen una lectura integrada de los códigos verbal y gráfico no demuestran diferencias entre lectores de diverso grado de inserción disciplinar, hecho que aparentemente se explica por una mayor carga cognitiva de procesamiento y por mayores requerimientos de la memoria de trabajo (Efecto General de Redundancia).

Desde una perspectiva teórica, estos hallazgos empíricos llevan a retomar la reflexión acerca de la naturaleza multisemiótica de los textos escritos estáticos y de los sistemas de codificación de información textual, así como de los múltiples códigos cognitivos requeridos para procesar dichos datos textuales. Adquiere, entonces, nuevamente relevancia el mencionado Principio de la Predominancia Constitutiva, en el marco de la Teoría de la Comunicabilidad, pues ya ha quedado establecido que sí se puede comprender un texto desde un único sistema predominante; claro está que en condiciones de conocimientos especializados y nivel universitario avanzado. Investigaciones contemporáneas como las de Schüler et al. (2015) y Rau et al. (2015) ponen de manifiesto la alta relevancia de profundizar en las teorías textuales de interacción entre los distintos sistemas semióticos y sus tipos y grados de conexión, considerando diversas variables tanto textuales como cognitivas.

En definitiva, la respuesta a nuestra pregunta ya instalada en el título de este estudio, es que sí se puede leer un texto especializado disciplinar desde la predominancia de un solo sistema semiótico, toda vez que los lectores sean integrantes avanzados de la comunidad y tengan un grado de inserción disciplinar, posean conocimiento del género IPOM y muestren manejo de los sistemas semióticos y los artefactos constitutivos.

De acuerdo con los datos presentados, los comprendedores de curso avanzado muestran recuperar en sus resúmenes los núcleos retórico/funcionales centrales de la movida retórica funcional constitutiva del pasaje textual en las versiones en que predomina un solo sistema semiótico, ya sea verbal o gráfico. No así necesariamente en la versión completa e integrada. Esto quiere decir que los resúmenes muestran que los lectores han comprendido - de modo importante- la Constatación, Evidencia y Proyección y que, según la TC, ellos logran acreditar lo comprendido respetando la organización retórica del texto fuente y dando muestras de ello en la construcción del resumen solicitado.

En cuanto a fortalezas, limitaciones y proyecciones, cabe señalar que esta investigación difiere de otros estudios similares en varios aspectos. Por ejemplo, nuestros materiales están basados en un estudio de corpus en la disciplina de economía 
y el género seleccionado corresponde a uno que circula en ámbitos académicos y profesionales (Corpus PUCV-UCSC-2013); al mismo tiempo, los textos de este género han sido estudiados y descritos en su organización retórica funcional y sus rasgos multisemióticos, hechos que nos permitieron seleccionar un segmento retórico y unos artefactos bien definidos y con características ecológicas o naturalísticas y situadas contextualmente. Además, la técnica del resumen ha resultado ser una alternativa eficiente de elicitación de la comprensión del texto y en coherencia con la Teoría de la Comunicabilidad.

Una limitación metodológica reside en no contar con una separación previa entre lectores de alto y bajo rendimiento en comprensión. Esta agrupación previa de los sujetos habría aportado información importante para establecer otra perspectiva de análisis, sobre todo en los estudiantes de primer año. En esta investigación, esta fase estaba contemplada en el diseño inicial, pero los resultados alcanzados no permitieron constituir dos grupos suficientemente independientes de lectores en cada nivel universitario. Ello conlleva, además, la necesidad de contar con una muestra más amplia de estudiantes en investigaciones futuras. Por otro lado, mayor análisis textual de la relaciones entre el sistema verbal y el sistema gráfico visual se requiere para poder calibrar de modo más profundo el tipo de integración de información textual y su correspondiente carga cognitiva de procesamiento, sobre todo en la versión completa integrada (Prueba A).

A pesar de estas limitaciones, el diseño de investigación parece suficientemente prometedor para abrir caminos a futuras investigaciones, tanto en lo relativo a los aspectos teóricos de, por ejemplo, número y formato de sistemas de representación multisemiótico tanto del texto como del sistema cognitivo, así como a otras vertientes más aplicadas e instruccionales, como el diseño de corpus para aprendientes. Como ha sido destacado por otros investigadores como Mayer (2009) y Schüler et al. (2015), coincidimos en sostener que las buenas teorías que aborden la comprensión multisemiótica requieren estar cimentadas en investigación cognitiva; a lo que agregamos que es fundamental también que esas investigaciones hagan converger estudios lingüísticos de corte tanto teórico como empírico. Esta integración resulta, sin duda, una dupla poderosa.

Pontificia Universidad Católica de Valparaíso* Avda. El Bosque 1290, Viña del Mar (Chile) gparodi@ucv.cl

Pontificia Universidad Católica de Valparaíso** Avda. El Bosque 1290, Viña del Mar (Chile) cristobal.julio.vergara@gmail.com 


\section{OBRAS CITADAS}

Ainsworth, Shaaron. "DeFT: A conceptual framework for considering learning with multiple representations". Learning and Instruction 16 (2006):183-198.

Alvermann, Donna, Unrau, Norman y Ruddell, Robert. (Eds.). Theoretical Models and Processes of Reading. Newark, NJ: IRA, 2013.

Aravena, Cristián. Artefactos multisemióticos en el género Artículo de Investigación de Lingüística y Química a nivel de doctorado. Ponencia presentada en el XIX Congreso Internacional de la Sociedad Chilena de Lingüística. Valparaíso, noviembre, 2011.

Arnoux, Elvira. La lectura y la escritura en la universidad. Buenos Aires: Eudeba, 2002.

Bateman, John. Text and Image: A Critical Introduction to the Visual/Verbal Divide. London: Routledge, 2014.

Boudon, Enrique y Parodi, Giovanni. "Artefactos multisemióticos y discurso académico de la economía: Construcción de conocimientos en el género Manual". Revista Signos. Estudios de Lingüística, 47(85) (2014):164-195.

Brünken, Roland, Steinbacher, Susan; Plass, Jan y Leutner, Detlev. "Assessment of cognitive load in multimedia learning using dual-task methodology". Experimental Psychology 49(2) (2002):109-119.

Coch, Cristoph. Consciousness. Confessions of a Romantic Reductionist. Cambridge: The MIT Press, 2012.

Chandler, Paul y Sweller, John. "Cognitive load theory and the format of instruction". Cognition and Instruction 8 (1991):293-332.

Dehaene, Stanislas. The Number Sense: How the Mind Creates Mathematics. Nueva York: Oxford University Press, 2011.

_ Reading in the Brain: The New Science of How We Read. Nueva York, Penguin Books, 2010.

De Vega, Manuel y Cuetos, Fernando. (Coords.). Psicolingüistica del Español. Madrid: Trotta, 1999.

De Vega, Manuel, Carreiras, Manuel, Gutiérrez-Calvo, Manuel y Alonso-Quecuty, Mauricio. Lectura y Comprensión: una Perspectiva Cognitiva. Madrid: Alianza, 1990.

De Vega, Manuel, Glenberg, Arthur y Graesser, Arthur. (Eds.) Symbols and Embodiment: Debates on Meaning and Cognition. Oxford: Oxford University Press, 2008.

Farías, Miguel, Obilinovic, Katica, Orrego, Roxana y Gregersen, Tammy. "Evaluating types and combinations of multimodal presentations in the retention and transfer of concrete vocabulary in EFL learning". Revista Signos. Estudios de Lingüística, 47(84) (2014):21-39. 
Fletcher, John y Tobias, Sigmund. "The Multimedia Principle”. En Richard, Mayer (Ed.), The Cambridge Handbook of Multimedia Learning. Cambridge: Cambridge University Press, 2005:117-133.

Gladic, Jadranka. Niveles de comprensión y su relación con la predominancia de sistemas semióticos en textos del área de la lingüistica: una aproximación al fenómeno multimodal desde el discurso académico universitario. Tesis para optar al Grado de Magíster en Lingüística Aplicada. Pontificia Universidad Católica de Valparaíso, Chile, 2012.

Graesser, Arthur, Singer, Murray y Trabasso, Tom. "Constructing inferences during narrative text comprehension". Psychological Review 101 (1994): 371-395.

Hiippala, Tuomo. "The interface between rhetoric and layout in multimodal artefacts". Literary and Linguistic Computing 28(3) (2012):461-471.

Holsanova, Jana. Discourse, Vision, and Cognition. Amsterdam: Benjamins, 2008.

Holsanova, Jana y Nord, Andreas. "Multimodal design: Media structures, media principles and users' meaning-making in printed and digital media". En Helmut, Bucher, Thomas, Gloning y Keneth, Lehnen (Eds.), Neue Medie - Neue Formate. Ausdifferenzierung und Konvergenz in der Medienkommunikatium. Frankfurt: Campus, 2010: 81-103.

Ibáñez, Romualdo. "Comprensión de textos académicos escritos en inglés: Relación entre nivel de logro y variables involucradas". Revista Signos. Estudios de Lingüistica 41(67) (2008):203-229.

Kintsch, Walter. Comprehension: A Paradigm for Cognition. Cambridge: Cambridge University Press, 1998.

Leahy, Wayne y Sweller, John. "Cognitive load theory, modality of presentation and the transient information effect". Applied Cognitive Psychology, 25(6) (2011):943-951.

Lemke, Jay. "Multiplying Meaning: Visual and Verbal Semiotics in Scientific Text". En James, Martin y Robert, Veel (Eds.), Reading Science. Londres: Routledge, 1998: $87-113$.

León, José. (Coord.). Conocimiento y Discurso. Claves para Inferir y Comprender. Madrid: Pirámide, 2003.

Louwerse, Max. "Symbolic or Embodied representations. A case of symbol interdependency". Topics in Cognitive Science, 2010:1-30.

Manghi, Dominique. "Géneros en la enseñanza escolar: Configuraciones de significado en clases de historia y biología desde una perspectiva multimodal". Revista Signos. Estudios de Lingüistica, 46(82)(2013):236-257.

Mayer, Richard. "Cognitive theory of multimedia learning". En Richard, Mayer (Ed.), The Cambridge Handbook of Multimedia Learning. Cambridge: Cambridge University Press, 2005:31-48.

— Multimedia Learning. Cambridge: Cambridge University Press, 2009. 
- “Applying the science of learning to multimedia instruction". En John, Mestre y Brian, Ross (Eds.), The Psychology of Learning and Motivation. Nueva York: Academic Press, 2011:77-108.

McCloskey, Deirdre. The Rhetoric of Economics. Madison: University of Wisconsin Press, 1985.

McNamara, Danielle y Magliano, Joseph. "Toward a Comprehensive Model of Comprehension". En Brian, Ross (Ed.), The Psychology of Learning and Motivation: Advances in Research and Theory. Illinois: Academic Press, 2009:297-372.

O'Halloran, Kay. Mathematical Discourse. Language, Symbolism and Visual Images. Londres: Continuum, 2006.

Otero, José, León, José y Graesser, Arthur. (Eds.). The Psychology of Science Text Comprehension. Mahwah, NJ: Erlbaum, 2002.

Parodi, Giovanni. Comprensión de textos escritos. La teoría de la comunicabilidad. Buenos Aires: Eudeba, 2014.

"La Teoría de la Comunicabilidad: Apuntes para una concepción integral de la comprensión de textos escritos". Revisa Signos. Estudios de Lingüística, 44(76) (2011):145-167.

_ "Multisemiosis y lingüística de corpus: Artefactos (multi)semióticos en los textos de seis disciplinas en el corpus PUCV-2010". Revista de Lingüistica Teórica y Aplicada (RLA) 48-2 (2010):33-70.

— "Comprensión y aprendizaje a partir del discurso especializado escrito: Teoría y empiria". En Giovanni, Parodi (Ed.), Lingüística de corpus y discursos especializados: Puntos de mira. Valparaíso: EUV, 2007:223-258.

_ Relaciones entre Lectura y Escritura: una Perspectiva Cognitiva Discursiva. Antecedentes Teóricos y Resultados Empíricos. Valparaíso: EUV, 2003.

Parodi, Giovanni, Julio, Cristóbal y Vásquez-Rocca, Liliana. "Los géneros del Corpus PUCV-UCSC-2013 del discurso académico de la economía: el caso del Informe de Política Monetaria”. Revista ALED, 15(3) (en prensa), 2015.

Parodi, Giovanni, Boudon, Enrique y Julio, Cristóbal. "La organización retórica del género Manual de economía: Un discurso en tránsito disciplinar". Revista de Lingüística Teórica y Aplicada (RLA), 52(2) (2014):133-163.

Parodi, Giovanni, Ibáñez, Romualdo y Venegas, René. “¿Cómo escribir un buen resumen?”. En Estrella, Montolío (Coord.), Manual de Escritura Académica y Profesional. Barcelona: Ariel, 2014:93-119.

Parodi, Giovanni y Burdiles, Gina. (Eds.). Leer y Escribir en Contextos Académicos y Profesionales: Géneros, Corpus y Métodos. Santiago de Chile: Ariel, 2015.

Parodi, Giovanni, Peronard, Marianne e Ibáñez, Romualdo. Saber Leer. Madrid: Aguilar, 2010.

Paivio, Allan. Mind and its Evolution: A Dual Coding Theoretical Approach. Mahwah, NJ: Erlbaum, 2007. 
_ Images in Mind: The Evolution of a Theory. Sussex, U.K.: Harvester Wheatsheaf, 1991.

— Mental Representation: A Dual Coding Approach. Nueva York: Oxford University Press, 1986.

_ Imagery and Verbal Processes. Nueva York: Holt, Rinehart \& Winston, 1971.

Plass, Jan, Moreno, Roxana y Brünken, Roland. (Eds.). Cognitive Load Theory. Cambridge: Cambridge University Press, 2010.

Rau, Martina, Michaelis, Joseph y Fay, Natalie. "Connection making between multiple graphical representations: A multi-methods approach for domain-specific grounding of an intelligent tutoring system for chemistry". Computer \& Education 82 (2015):460-485.

Royce, Terry. Visual-verbal intnersemiotic complementarity in the Economist magazine. Tesis doctoral, The University of Reading, Reading, Inglaterra, 1999.

Rummer, Ralf, Schweppe, Judith, Fürstenberg, Anne, Scheiter, Katharina y Zindler, Antje. "The perceptual basis of the modality effect in multimedia learning". Journal of Experimental Psychology: Applied 17 (2011):159-173.

Sadoski, Mark. "Imagination, cognition, and persona". Rhetoric Review, 10, 266-278, 1992.

Sadoski, Mark y Paivio, Allan. Imagery and text: A dual coding theory of reading and writing. Mahwah, NJ: Erlbaum, 2001.

_ “ "Toward a unified theory of reading". Scientific Studies of Reading 11 (2007):337356.

Sadoski, Mark, Paivio, Allan y Goetz, Ernest. "A critique of schema theory in reading and a dual coding alternative". Reading Research Quarterly, 26 (1991):463-484.

Sadoski, Mark y Willson, Victor. "Effects of a theoretically based large-scale reading intervention in a multicultural urban school district". American Educational Research Journal, 43 (2006):137-154.

Sadoski, Mark; Willson, Victor, Holcomb, Angelia y Boulware-Gooden, Regina. "Verbal and nonverbal predictors of spelling performance". Journal of Literacy Research 36 (2005):461-478.

Sanchez, Cristopher y Wiley, Jennifer. "The role of dynamic spatial ability in geoscience text comprehension". Learning and Instruction 31 (2014): 33-45.

Santana, Andrea. Nivel de dominio del inglés, multimodalidad y nivel de comprensión en segunda lengua de textos académicos escritos en inglés: una aproximación al fenómeno en el ámbito de la Física. Tesis para optar al grado de Magíster en Lingüística Aplicada, Pontificia Universidad Católica de Valparaíso, Chile, 2010.

Segers, Eliane, Verhoeven, Ludo y Hulstijn-Hendrikse, Nanneke. "Cognitive processes in children's multimedia text learning". Applied Cognitive Psychology 22 (2008): 375-387.

Schnotz, Wolfgang. "An integrated model of text and picture comprehension". En Richard, Mayer (Ed.), The Cambridge Handbook of Multimedia Learning. Cambridge: Cambridge University Press, 2005:49-69. 
- "Towards an Integrated View of Learning from Text and Visual Displays". Educational Psychology Review, 14(2) (2002):101-120.

Schnotz, Wolfgang y Bannert, Maria. "Construction and interference in learning from multiple representations". Learning and Instruction 13, (2003):141-156.

Schnotz, Wolfgang, Bannert, Maria y Seufert, Thomas. "Towards an integrative view of text and picture comprehension: Visualization effects on the construction of mental models". En Arthur, Graesser, José, Otero y José Antonio, León (Eds.), The Psychology of Science Text Comprehension. Mahwah, NJ: Erlbaum, 2002:385416.

Schnotz, Wolfgang y Horz, Holger. "New Media, Learning from”. En Elizabeth, Baker, Patrick, Peterson \& Ben, McGaw (Eds.), International Encyclopedia of Education. Nueva York: Elsevier, 2010:140-149.

Schüler, Anne, Arndt, Jana y Scheiter, Katharina. "Processing multimedia material: Does integration of text and pictures result in a single or two interconnected mental representations?" Learning and Instruction 35 (2015):62-72.

Sweller, John. "Implications of Cognitive Load Theory for Multimedia Learning". En Richard, Mayer (Ed.), The Cambridge Handbook of Multimedia Learning. Cambridge: Cambridge University Press, 2005:19-30.

_ "Evolution of human cognitive architecture". En Brian, Ross (Ed.), The Psychology of Learning and Motivation. San Diego, CA: Academic Press, 2003:215-216.

__ Instructional design in technical areas. Camberwell, Australia: ACER Press, 1999. "Cognitive load during problem solving: Effects on learning". Cognitive Science, 12 (1988):257-285.

Sweller, John, Ayres, Paul y Kalyuga, Slava. (Eds.). Cognitive Load Theory. Nueva York: Springer, 2011.

Taboada, Maite y Habel, Christopher. "Rhetorical relations in multimodal documents". Discourse Studies, 15(1) (2013):65-89.

Van Essen, David y Dieker, Donna. "Surface-based and probabilistic atlases of primate cerebral cortex". Neuron, 56 (2007):209-224.

Van Dijk, Teun y Kintsch, Walter. Strategies of Discourse Comprehension. Nueva York: Academic Press, 1983.

Van Leeuwen, Theo. Typographic meaning. Visual Communication, 4(2) (2005):137143.

— Towards a semiotics of typography. Information Design Journal + Document Design, 4(2) (2006):139-155.

— The Language of Colour. An Introduction. Londres: Routledge, 2011.

Vásquez, Liliana. Conexiones entre artefactos multisemióticos y organización retórica en la construcción de significado en el género IPOM del discurso de la economía. Ponencia presentada en el VIII Encuentro Nacional de Estudios del discurso, ALED Chile. Organizado por la Universidad de Valparaíso, Viña del Mar, Noviembre, 2014. 